\title{
A Commingle between the Contractive Conditions of Type Jungck and a General Weak Commutativity Concept
}

\author{
Salvatore Sessa ${ }^{1, *,+(\mathbb{D}, \text { Waleed M. Alfaqih }}{ }^{2,+}+\mathbb{C}$ and Mohammad Imdad ${ }^{3,+}$ \\ 1 Dipartimento di Architettura, Università degli Studi di Napoli Federico II, Via Toledo 402, \\ 80134 Napoli, Italy \\ 2 Department of Mathematics, Hajjah University, Hajjah, Yemen; waleedmohd2016@gmail.com \\ 3 Department of Mathematics, Aligarh Muslim University, Aligarh 200201, India; mimdad.mm@amu.ac.in \\ * Correspondence: sessa@unina.it \\ $\dagger$ These authors contributed equally to this work.
}

Received: 26 June 2020; Accepted: 23 July 2020; Published: 26 July 2020

check for updates

\begin{abstract}
In this work, we prove a common fixed point theorem of type Jungck under a generalized definition of weak commutativity and a well-known contractive inequality by commingling both conditions in the proof. Some open questions are also indicated and intimately connected (or not?) to the metric to be used.
\end{abstract}

Keywords: wc pair; co pair; wco pair; owcpair; general weak commutativity

\section{Introduction}

In 1976, Jungck [1] introduced the notion of commutativity of two mappings $f$ and $h$ and utilized the same to prove the existence of a common fixed point. This notion has been improved by Sessa $[2,3]$ in which he introduced the notion of weak commutativity and provide a common fixed point results for a pair $(f, h)$ of self-mappings defined on a complete metric space. Thereafter, Jungck [4] introduced the very useful notion namely compatibility of mappings and utilized this notion to prove common fixed points for certain mappings. As a result, several researchers improved the concept of compatibility of mappings by introducing weaker versions of this concept such as weak compatibility and occasionally weakly compatibility (cfr., e.g., [5-10]). In this regard, a flood of common fixed point results have been offered by various researchers utilizing several types of commutativity of mappings.

The aim of this paper is to introduce a new generalization of the notion of weak commutativity by commingling the same with the well-known contractive inequality in the proof. We utilize our newly introduced notions to prove a common fixed point theorem of type Jungck. Some open questions are also indicated and intimately connected (or not?) to the metric to be used.

\section{Definitions and General Concepts}

Throughout this work, $n$ denotes for a positive integer and $\mathbb{R}$ is the set of all real numbers.

We recall some well-known definitions in the metric fixed point theory. Let $f$ and $h$ be two self-maps of a metric space $(X, d)$. Then $C(f, h):=\{\aleph \in X: f(\aleph)=h(\aleph)\}$, i.e., the set of all coincidence points of $f$ and $h$.

The pair $(f, h)$ is said to be:

1. Weakly commuting (wc) [2] if

$$
d(f(h(\aleph)), h(f(\aleph))) \leq d(f(\aleph), h(\aleph)) \forall \aleph \in X .
$$


2. Compatible (co) [3] if $\exists\left\{\aleph_{n}\right\} \subseteq X$ satisfying

$$
\lim _{n \rightarrow \infty} d\left(f\left(h\left(\aleph_{n}\right)\right), h\left(f\left(\aleph_{n}\right)\right)\right)=0 \text { whenever } \lim _{n \rightarrow \infty} f\left(\aleph_{n}\right)=\lim _{n \rightarrow \infty} h\left(\aleph_{n}\right)=\mathrm{M} \text { for some } \mathrm{M} \in X
$$

3. Weakly compatible (wco) [4] if

$$
f(h(\aleph))=h(f(\aleph)) \forall \aleph \in C(f, h) .
$$

4. Occasionally weakly compatible (owc) (cfr., e.g., [5-11]) if

$$
f(h(\aleph))=h(f(\aleph)) \text { for } \aleph \in C_{0}(f, h):=\{\aleph \in C(f, h): f(h(\aleph))=h(f(\aleph))\} .
$$

Remark 1. The following implications hold:

$$
w c \Longrightarrow w c o \Longrightarrow o w c,
$$

but the converse generally does not hold as shown in [6].

The following example (borrowed from [6]) proves that the class of wco maps is a proper subclass of the class of owc maps.

Example 1. Let $X=[0, \infty)$ be equipped with the Euclidean metric. Let us define $f, h: X \rightarrow X$ by

$$
f(\aleph)=2 \aleph \text { and } h(\aleph)=\aleph^{2} \forall \aleph \in X .
$$

It is easy to show that $C(f, h)=\{0,2\}$. Observe that

$$
f(h(0))=h(f(0)) \text { and } 8=f(h(2)) \neq h(f(2))=16 .
$$

Thus, $(f, h)$ is an owc pair but it is neither wco nor wc because

$$
d(f(h(\aleph)), h(f(\aleph)))=\left|2 \aleph^{2}-4 \aleph^{2}\right|=2 \aleph^{2}>\aleph|\aleph-2|=d(f(\aleph), h(\aleph)), \forall \aleph \geq 2 .
$$

Remark 2. In [6] the authors proved that in the setting of a discrete metric spaces the pair $(f, h)$ is wc iff it is wco.

Now, we introduce a new definition as follows:

Definition 1. The pair $(f, h)$ is said to be generally weakly commuting $(g w c)$ if

$$
d(f(h(\aleph)), h(f(\aleph))) \leq \max \{d(f(\aleph), h(\aleph)), d(f(h(\aleph)), h(\aleph)), d(h(f(\aleph)), f(\aleph))\}
$$

for all $\aleph \in X$.

In the following proposition, we prove that in the setting of discrete metric spaces owc implies gwc.

Proposition 1. Let $(X, d)$ be a discrete metric space and $(f, h)$ a non-trivial owc pair defined on $X$. Then $(f, h)$ is also a gwc pair.

Proof. Given that $(f, h)$ is a non-trivial owc pair defined on $X$, so $C_{0}(f, h) \neq \phi$. Observe that for any $\aleph \in C_{0}(f, h)$ we have $d(f(\aleph), h(\aleph))=d(f(h(\aleph)), h(f(\aleph)))=0$. Now, if $\aleph$ is not in $C_{0}(f, h)$, 
but $\aleph$ is in $C(f, h)$, then $f(h(\aleph)) \neq h(f(\aleph))$ and $f(\aleph)=h(\aleph)$. Hence, $d(f(h(\aleph)), h(f(\aleph)))=1$ and $d(f(\aleph), h(\aleph))=0$. So, on using triangular inequality, we have

$$
1=d(f(h(\aleph)), h(f(\aleph))) \leq d(f(h(\aleph)), h(\aleph))+d(h(\aleph), h(f(\aleph)))
$$

which implies that either $d(f(h(\aleph)), h(\aleph))=1$ or $d(h(\aleph), h(f(\aleph)))=1$, hence

$$
1 \leq \max \{d(f(h(\aleph)), h(\aleph)), d(h(\aleph), h(f(\aleph))), 0\}=1 .
$$

If $\aleph$ is not in $C(f, h)$, then

$$
f(\aleph) \neq h(\aleph), \text { i.e., } d(f(\aleph), h(\aleph))=1,
$$

and either

$$
f(h(\aleph))=h(f(\aleph), \text { i.e., } d(f(h(\aleph)), h(f(\aleph)))=0,
$$

or

$$
d(f(h(\aleph)), h(f(\aleph)))=1, \text { i.e., } f(h(\aleph)) \neq h(f(\aleph) .
$$

In the both cases, we have

$$
d(f(h(\aleph)), h(f(\aleph))) \leq \max \{1, d(f(h(\aleph)), h(\aleph)), d(h(f(\aleph)), f(\aleph))\}=1 .
$$

Therefore, $(f, h)$ is a gwc pair.

Remark 3. If $C(f, h)=\phi$, and hence $C_{0}(f, h)=\phi$, then $(f, h)$ is trivially a gwc pair.

The following example is fitting to Definition 1.

Example 2. Let $X=[0, \infty)$ be equipped with the discrete metric. Define $f$ and $h$ as in Example 1. So $(f, h)$ is an owc pair because $C_{0}(f, h)=\{0\}$. Notice that

$$
d(f(2), h(2))=0<1=d(f(h(2)), h(f(2))),
$$

i.e., the pair $(f, h)$ is not a wo pair.

Since $f(h(\aleph))=2 \aleph^{2}, h\left(f(\aleph)=4 \aleph^{2}\right.$ for any $\aleph \in X$, we have:

- $f \circ r \aleph \in-\{0,2\}$,

$$
1=d(f(h(\aleph)), h(f(\aleph)))=\max \{d(f(\aleph), h(\aleph)), d(f(h(\aleph)), h(\aleph)), d(h(f(\aleph)), f(\aleph))\}=\max \{1,1,1\},
$$

- $\quad$ for $\aleph=2$,

$$
1=d(f(h(2)), h(f(2))=\max \{d(f(2), h(2)), d(f(h(2)), h(2)), d(h(f(2)), f(2))\}=\max \{0,1,1\},
$$

- $\quad$ for $\aleph=0$,

$$
0=d(f(h(0)), h(f(0)))=\max \{d(f(0), h(0)), d(f(h(0)), h(0)), d(h(f(0)), f(0))\}=\max \{0,0,0\} .
$$

Hence, $(f, h)$ is a gwc pair.

Observe that if $d$ is the Euclidean metric, we have

$$
d(f(h(\aleph)), h(f(\aleph)))=2 \aleph^{2}
$$




$$
\begin{gathered}
d(f(h(\aleph)), h(\aleph))=\aleph^{2}, \\
d(h(f(\aleph)), f(\aleph))=3 \aleph^{2}, \\
d(f(\aleph), h(\aleph))=\left|\aleph^{2}-2 \aleph\right| .
\end{gathered}
$$

Thus, $(f, h)$ is also gwc since

$$
2 \aleph^{2} \leq 3 \aleph^{2} \leq \max \left\{\left|\aleph^{2}-2 \aleph\right|, 3 \aleph^{2}, \aleph^{2}\right\} \text { for any } \aleph \in X
$$

Question 1. Besides the discrete metric, under which other metrics, semi-metrics, b-metrics, ..., we can guarantee the equivalence between the concepts of owc and gwc? Either these concepts prescind from the metric used or they are strongly dependent from the metric in accordance to the problem under exam?

We explicitly observe that the following theorem holds:

Theorem 1. [3] Let $f$ and $h$ be two continuous self-maps of a metric space $(X, d)$. If $f$ is a proper map (i.e., $f^{-1}(C)$ is compact subset of $X$ whenever $C$ is compact subset of $\left.X\right)$, then $(f, h)$ is a co pair iff it is an owc pair.

In favour of this result, we give the following example:

Example 3. Let $X=\mathbb{R}$ be equipped with the Euclidean metric. Define $f$ and $h$ by

$$
f(\aleph)=\aleph^{2} \text { and } h(\aleph)=\aleph^{3}-\aleph \text { for any } \aleph \in \mathbb{R} \text {. }
$$

It is not difficult to verify that both $f$ and $h$ are proper. Solving the equation $\aleph\left(\aleph^{2}-1\right)=\aleph^{2}$, one deduces that

$$
C(f, h)=\left\{0, \frac{1+\sqrt{5}}{2}, \frac{1-\sqrt{5}}{2}\right\}
$$

Notice that $\frac{1+\sqrt{5}}{2}$ and $\frac{1-\sqrt{5}}{2}$ are the opposite of the negative and positive golden ratios, respectively, and $f(0)=h(0)=0, f\left(\frac{1 \mp \sqrt{5}}{2}\right)=h\left(\frac{1 \mp \sqrt{5}}{2}\right)=\frac{3 \mp \sqrt{5}}{2}$.

Observe that

$$
f(h(\aleph))=\aleph^{6}+\aleph^{2}-2 \aleph^{4}=\aleph^{6}-\aleph^{2}=h(f(\aleph)) \Longleftrightarrow \aleph \in\{-1,0,1\} .
$$

Hence, $C_{0}(f, h)=\{0\}$. Therefore, $(f, h)$ is an owc pair. Notice that it is possible to consider infinite sequences $\left\{\aleph_{n}\right\}$ converging to 0 (among the others, $\aleph_{n}=0$ for any $n$ ) such that

$$
\lim _{n \rightarrow \infty} f\left(\aleph_{n}\right)=\lim _{n \rightarrow \infty} h\left(\aleph_{n}\right)=\lim _{n \rightarrow \infty} d\left(f\left(h\left(\aleph_{n}\right)\right), h\left(f\left(\aleph_{n}\right)\right)\right)=\lim _{n \rightarrow \infty} 2 \aleph_{n}^{2}\left|\aleph_{n}^{2}-1\right|=0
$$

\section{A Commingle Inside a Common Fixed Point Theorem}

To establish a priori that $C(f, h) \neq \phi$ or $C_{0}(f, h) \neq \phi$ is a wishful thinking (sometimes directly unrealistic) because it could be difficult to solve the equation $f(h(\aleph))=h(f(\aleph))$ ex abrupt from which to obtain $f(\aleph)=h(\aleph)$ necessarily. Instead to verify a general weak commutativity concept under a metric in an adequate environment (such as a metric space or a Banach space, ...) could be more easy when one tries to theorize common fixed point theorems of type Jungck [1] for related applications. An example of theorem is given in the sequel by strengthening slightly the above definition of gmc, practically showing as a commingle between the contractive condition of type Jungck and a certain definition of type gmc can imply the existence of common fixed points for a pair $(f, h)$. 
First of all, we strength a little bit the previous definition of gmc with the following:

Definition 2. The pair $(f, h)$ is called quasi-generally weakly commuting ( $q g w c)$ if

$$
d(f(h(\aleph)), h(f(\aleph))) \leq d(f(\aleph), h(\aleph))+\frac{1}{2} \max \{d(f(h(\aleph)), h(\aleph)), d(h(f(\aleph)), f(\aleph))\},
$$

for all $\aleph \in X$.

Then the following theorem holds:

Theorem 2. Let $(f, h)$ be a qgwc pair of self-maps of a metric space $(X, d)$. Assume that $C(f, h) \neq \phi$ and there exists a constant $k<1$ such that

$$
d(f(\aleph), f(\mathrm{M})) \leq k d(h(\aleph), h(\mathrm{M})) \text { for all } \aleph, \mathrm{M} \in X .
$$

Then $C_{0}(f, h) \neq \phi$ and $f$, h have a unique common fixed point.

Proof. Let $\aleph \in C(f, h)$, then the next inequalities are a commingle between the qgwc property and the contractive condition. Indeed, we have

$$
d(f(h(\aleph)), h(\aleph))=d(f(f(\aleph)), f(\aleph)) \leq k d(h(f(\aleph)), h(\aleph))=k d(h(f(\aleph)), f(\aleph))
$$

and hence (by qgwc property)

$$
d(f(h(\aleph)), h(f(\aleph))) \leq 0+\frac{k}{2} d(h(f(\aleph)), f(\aleph))
$$

which implies (by triangular inequality)

$$
2 d(f(h(\aleph)), h(f(\aleph))) \leq k d(h(f(\aleph)), f(\aleph)) \leq k(d(h(f(\aleph)), f(h(\aleph)))+d(f(\aleph), f(h(\aleph)))
$$

and then

$$
\begin{aligned}
(2-k) d(f(h(\aleph)), h(f(\aleph))) & \leq k d(f(\aleph), f(h(\aleph)))=k d(h(\aleph), f(h(\aleph))) \\
& \leq k^{2} d(h(f(\aleph)), f(\aleph)) \\
& \leq k^{2}(d(h(f(\aleph)), f(h(\aleph)))+d(f(\aleph), f(h(\aleph)))
\end{aligned}
$$

which implies

$$
\left(2-k-k^{2}\right) d(f(h(\aleph)), h(f(\aleph))) \leq k^{2} d(f(\aleph), f(h(\aleph))) .
$$

Thus, iterating we have that

$$
\left(2-k-k^{2}-\ldots-k^{n}\right) d(f(h(\aleph)), h(f(\aleph))) \leq k^{n} d(f(\aleph), f(h(\aleph))),
$$

that is

$$
\left(3-\left(1+k+k^{2}+\ldots+k^{n}\right)\right) d(f(h(\aleph)), h(f(\aleph))) \leq k^{n} d(f(\aleph), f(h(\aleph)))
$$

that is

$$
\left(3-\frac{1-k^{n+1}}{1-k}\right) d(f(h(\aleph)), h(f(\aleph))) \leq k^{n} d(f(\aleph), f(h(\aleph))) .
$$

By putting $n \rightarrow \infty$, finally we have

$$
\left(3-\frac{1}{1-k}\right) d(f(h(\aleph)), h(f(\aleph)))=0 .
$$


Then

$$
f(h(\aleph))=h(f(\aleph))=h(h(\aleph))=f(f(\aleph))
$$

and hence $\aleph \in C_{0}(f, h)$. Moreover,

$$
d(f(f(\aleph)), f(\aleph)) \leq k d(h(f(\aleph)), h(\aleph))=k d(f(f(\aleph)), f(\aleph)),
$$

therefore $z=f \aleph$ is a fixed point of $f$. Furthermore,

$$
h(z)=h(f(\aleph))=f(f(\aleph))=f(z)=z
$$

and thus $z$ is the common fixed point of $f$ and $h$. Now, if $w$ is another common fixed point, then $d(z, w)=d(f(z), f(w)) \leq k d(h(z), h(w))=k d(z, w)$, which implies $z=w$.

Question 2. It is an open question to establish that Theorem 2 holds under the above concept of gmc pair.

We illustrate Theorem 2 with the following example.

Example 4. Let $X=[1, \infty)$ be endowed with the Euclidean metric. Define $f$ and $h$ as

$$
f(\aleph)=\frac{\aleph+2}{3} \text { and } h(\aleph)=\aleph^{2} \text { for all } \aleph \in X .
$$

We have that

$$
\frac{1}{3}|(\aleph+2)-(M+2)|=\frac{1}{3}|\aleph-M| \leq \frac{1}{2}|\aleph-M||\aleph+M|,
$$

i.e.,

$$
d(f(\aleph), f(\mathrm{M})) \leq \frac{1}{2} d(h(\aleph), h(\mathrm{M})) \text { for all } \aleph, \in X .
$$

Furthermore, $C(f, h)=\{1\}$. Observe that

$$
\begin{gathered}
d(f(h(\aleph)), h(f(\aleph)))=\left|\frac{\aleph 2+2}{3}-\frac{(\aleph+2)^{2}}{9}\right|=\left|\frac{2 \aleph^{2}-4 \aleph+2}{9}\right|=\frac{2}{9}(\aleph-1)^{2}, \\
d(f(h(\aleph)), h(\aleph))=\frac{2 \aleph^{2}}{3}-\frac{2}{3}=\frac{2}{3}(\aleph 2-1), \\
d(h(f(\aleph)), f(\aleph))=\frac{|\aleph 2+4 \aleph+4-3 \aleph-6|}{9}=\frac{(\aleph 2+\aleph-2)}{9}=\frac{1}{9}(\aleph-1)(\aleph+2)
\end{gathered}
$$

and

$$
d(f(\aleph), h(\aleph))=\frac{|3 \aleph 2-\aleph-2|}{3}=\frac{|3 \aleph 2-\aleph-2|}{3}=\frac{(\aleph-1)(3 \aleph+2)}{3}
$$

for $\aleph \geq 1$. So, we have (for $\aleph \geq 1$ )

$$
\begin{aligned}
d(h(f(\aleph)), f(h(\aleph))) \mid & =\frac{2}{9}(\aleph-1)^{2} \leq \frac{1}{3}\left(\aleph^{2}-1\right) \\
& =\frac{1}{2} \frac{2}{3}\left(\aleph^{2}-1\right) \\
& =\frac{1}{2} d(f(h(\aleph)), h(\aleph)) \\
& \leq \frac{1}{2} \max \{d(f(h(\aleph)), h(\aleph)), d(h(f(\aleph)), f(\aleph))\}
\end{aligned}
$$


Also, we have (for $\aleph \geq 1$ )

$$
d(h(f(\aleph)), f(h(\aleph))) \mid=\frac{2}{9}(\aleph-1)^{2} \leq \frac{(\aleph-1)(3 \aleph+2)}{3}=d(f(\aleph), h(\aleph)) .
$$

Therefore, $f, h$ are also wc. Then, all the assumptions of Theorem 2 are satisfied. Hence, $C_{0}(f, h)=\{1\}$ which is the unique common fixed point of $f$ and $h$.

The next example is more instructive.

Example 5. Let $X=\{0\} \cup[4, \infty)$ and $f \aleph=\aleph^{2}$ and $h \aleph=\aleph^{3}-\aleph$ (cfr. Example 3). Then (for $\left.2 \leq \aleph\right)$

$$
d(f(h(\aleph)), h(f(\aleph)))=2 \aleph 4-2 \aleph 2>\aleph\left(\aleph^{2}-1-\aleph\right)=d(f(\aleph), h(\aleph))
$$

because the following implications hold ( for $4 \leq \aleph)$ :

$$
2 \aleph^{4}-2 \aleph^{2}>\aleph\left(\aleph^{2}-1 \aleph\right), \aleph^{3}-\aleph>\frac{\aleph^{2}-1-\aleph}{2}, \aleph^{3}-\frac{\aleph}{2}>\aleph^{2}-\frac{1}{2}, 2 \aleph^{3}-\aleph>\aleph^{2}-1 .
$$

Hence, $f$ and $h$ are not wc but they are qgwc as (for all $\aleph \in \aleph$ )

$$
\begin{aligned}
d(f(h(\aleph)), h(f(\aleph))) & =2 \aleph^{4}-2 \aleph^{2} \\
& \leq \frac{\aleph^{6}-2 \aleph^{2}}{2} \\
& =\frac{d(f(h(\aleph)), h(\aleph))}{2} \\
& \leq \frac{1}{2} \max \{d(f(h(\aleph)), h(\aleph)), d(h(f(\aleph)), f(\aleph))\}
\end{aligned}
$$

because the following implications hold (for all $\aleph \in X$ )

$$
\aleph^{4}-\aleph^{2} \leq \frac{\aleph^{6}-2 \aleph^{2}}{4}, 4 \aleph^{4}-4 \aleph^{2} \leq \aleph^{6}-2 \aleph^{2}, 4 \aleph^{2}-4 \leq \aleph^{4}-2,4 \aleph^{2} \leq \aleph^{4}+2 .
$$

The contractive condition of Theorem 2 is verified. Indeed, we assume $4 \leq \aleph<\mathrm{M}$ without loss of generality. Being $1 \leq \aleph \mathrm{M}$ and $\aleph^{2}+\mathrm{M}^{2}-1>0$, we have that

$$
(\aleph+M) \leq \aleph M(\aleph+M) \leq \frac{\aleph 2+M 2-1}{3}+\aleph^{2} M+\aleph M^{2} \leq \frac{\aleph^{2}+3 \aleph^{2} M+3 \aleph M^{2}+M^{2}-1}{3}
$$

and hence

$$
d(f(\aleph), f(\mathrm{M}))=\left(\aleph^{2}-\mathrm{M}^{2}\right) \leq\left(\aleph^{3}-\mathrm{M}^{3}\right)-\frac{\aleph-\mathrm{M}}{3}=\frac{d(h(\aleph), h(\mathrm{M}))}{3} .
$$

Moreover, for $\aleph=0$ and $4 \leq \mathrm{M}$ we have that

$$
d(f(0), f(\mathrm{M}))=\mathrm{M}^{2} \leq \frac{\mathrm{M}^{3}-\mathrm{M}}{3}=\frac{d(h(\aleph), h(\mathrm{M}))}{3} .
$$

Of course 0 is the unique common fixed point of $f$ and $h$. Note that in this case we have that $C(f, h)=C_{0}(f, h)=\{0\}$.

At this point fixed point theorems of type Jungck [1] to be established and deduced from Theorem 2 have right of citizenship in the worldwide and sparse literature of metrical fixed point theory. 


\section{Conclusions}

We show that the contractive condition and the general weak commutativity definition can be combined together for assuring a common fixed point theorem and we have illustrated this in Theorem 2 in the simplest possible manner (with a Banach type inequality) by leaving to the readers any feasible generalization. Any replies (even if partial) to the two above open questions are also welcome.

Author Contributions: All the authors contributed equally and significantly in writing this article. All authors have read and agreed to the published version of the manuscript.

Funding: This research was funded by the Department of the first author.

Acknowledgments: All the authors are grateful to the anonymous referees for their excellent suggestions, which greatly improved the presentation of the paper.

Conflicts of Interest: The authors declare no conflict of interest.

\section{References}

1. Jungck, G. Commuting mappings and fixed points. Amer. Math. Mon. 1976, 83, 261-263. [CrossRef]

2. Sessa, S. On a weak commutativity condition of mappings in fixed point considerations. Publ. Inst. Math. 1982, 32, 149-153.

3. Jungck, G. Common fixed points for commuting and compatible maps on compacta. Proc. Amer. Math. Soc. 1988, 103, 977-983. [CrossRef]

4. Jungck, G. Compatible mappings and common fixed points. Int. J. Math. Math. Sci. 1986, 9, 771-779. [CrossRef]

5. Al-Thagafi, M.A.; Shahzad, N. Generalized I-nonexpansive selfmaps and invariant approximations. Acta Math. Sin. Engl. Ser. 2008, 24, 867-876. [CrossRef]

6. Al-Thagafi, M.A.; Shahzad, N. A note on occasionally weakly compatible maps. Int. J. Math. Anal. 2009, $3,55-58$.

7. Abbas, M.; Rhoades, B.E. Common fixed point theorems for hybrid pairs of occasionally weakly compatible mappings satisfying generalized contractive condition of integral type. Fixed Point Theory Appl. 2007, 2007, 054101. [CrossRef]

8. Abbas, M.; Rhoades, B.E. Common fixed point theorems for hybrid pairs of occasionally weakly compatible mappings defined on symmetric spaces. Panam. Math. J. 2008, 2008, 35-54.

9. Jungck, G.; Rhoades, B.E. Fixed point theorems for occasionally weakly compatible mappings. Fixed Point Theory 2006, 7, 287-296.

10. Cho, Y.J. Survey on metric fixed point theory and applications. In Advances in Real and Complex Analysis with Applications; Springer: Berlin/Heidelberg, Germany, 2017; pp. 183-241

11. Imdad, M.; Sharma, A.; Chauhan, S. Unifying a multitude of common fixed point theorems in symmetric spaces. Filomat 2014, 28, 1113-1132. [CrossRef]

(C) 2020 by the authors. Licensee MDPI, Basel, Switzerland. This article is an open access article distributed under the terms and conditions of the Creative Commons Attribution (CC BY) license (http:/ / creativecommons.org/licenses/by/4.0/). 